\title{
O PAPEL DO FUNDO CONSTITUCIONAL DE FINANCIAMENTO DO NORTE E DO PROGRAMA NACIONAL DE FORTALECIMENTO DA AGRICULTURA FAMILIAR PARA A REGIÃO NORTE DO BRASIL
}

\author{
Gisalda Carvalho Filgueiras'; Elisabeth dos Santos Bentes ${ }^{2}$; André Cutrim Carvalho ${ }^{3}$; Ana \\ Cláudia de Sousa Araújo ; Clayton Douglas Chagas de Oliveira 5 . \\ ${ }^{1}$ Universidade Federal do Pará (UFPA), Belém, Pará, Brasil, gisaldaf@yahoo.com.br \\ 2 Universidade da Amazônia, Belém, Pará, Brasil, esbentes@gmail.com \\ ${ }^{3}$ UFPA, Belém, Pará, Brasil, andrecc83@gmail.com \\ ${ }^{4}$ UFPA, Belém, Pará, Brasil, nana22araujo@gmail.com \\ ${ }^{5}$ Universidade Federal do Sul e Sudeste do Pará, Marabá, Pará, Brasil, douglaslenoir@yahoo.com.br
}

\begin{abstract}
RESUMO: Esta pesquisa teve por objetivo analisar a aplicação dos recursos do crédito rural na Agricultura Familiar (AF) da região Norte. Admitiu-se que esses financiamentos têm contribuído para o crescimento da produção agropecuária da Região, tendo papel relevante na produção de alimentos, bem como na geração de renda e política de fixação do homem no campo. Os resultados revelaram que cada Estado possui dinâmica própria no que se refere ao setor rural, com maior ou menor grau de produção, dependendo da estrutura agrária e da atuação das políticas públicas direcionadas para aquele segmento; alguns estados têm baixa demanda do crédito Fundo Constitucional de Financiamento do Norte (FNO) e do Programa Nacional de Fortalecimento da Agricultura Familiar (PRONAF) por razões como conflitos agrários e engessamento das atividades do meio rural decorrente de Leis relativas à preservação ambiental; há concentração dos recursos concedidos para a expansão da produção da AF em poder de grupos com maior nível de renda e, somente alguns Estados são especializados no setor agropecuário.
\end{abstract}

PALAVRAS-CHAVE: Agricultura Familiar, Crédito Rural, Região Norte.

\section{THE ROLE OF THE NORTH FUNDING CONSTITUTIONAL FUND AND THE NATIONAL PROGRAM FOR STRENGTHENING FAMILY AGRICULTURE FOR THE NORTHERN REGION OF BRAZIL}

ABSTRACT: This research aimed to analyze the implementation of rural credit resources FNO Pronaf in agriculture/Familiar (AF) in the northern region. He admitted that these funds have contributed to the growth of agricultural production in the region, having a relevant role in food production, as well as in the generation of income and pricing policy in the field. The results revealed that: each State has its own dynamics with regard to the rural sector, with a greater or lesser degree, depending on the agrarian structure and the performance of public policies directed to that segment; some States have low credit demand of FNO/Pronaf for reasons as agrarian conflicts and casting of rural activities 
arising from laws relating to environmental protection; There are concentration of resources granted for the expansion of the production of the AF held by groups with higher income levels, and only some States specialize in the agricultural sector.

KEYWORDS: Family Agriculture, North Region, Rural Credit.

\section{EL PAPEL DEL FONDO CONSTITUCIONAL DE FINANCIAMIENTO DEL NORTE Y DEL PROGRAMA NACIONAL DE FORTALECIMIENTO DE LA AGRICULTURA FAMILIAR PARA LA REGIÓN NORTE DE BRASIL}

RESUMEN: Esta investigación tuvo por objetivo analizar la aplicación de los recursos del crédito rural en la Agricultura Familiar (AF) de la región Norte. Se admitió que estas financiaciones han contribuido al crecimiento de la producción agropecuaria de la Región, teniendo un papel relevante en la producción de alimentos, así como en la generación de renta y política de fijación del hombre en el campo. Los resultados revelaron que cada Estado posee dinámica propia en lo que se refiere al sector rural, con mayor o menor grado de producción, dependiendo de la estructura agraria y de la actuación de las políticas públicas dirigidas hacia ese segmento; Algunos estados tienen baja demanda del crédito Fondo Constitucional de Financiamiento del Norte (FNO) y del Programa Nacional de Fortalecimiento de la Agricultura Familiar (PRONAF) por razones como conflictos agrarios y enyesado de las actividades del medio rural derivado de Leyes relativas a la preservación ambiental; Hay una concentración de los recursos concedidos para la expansión de la producción de la AF en poder de grupos con mayor nivel de ingresos y, sólo algunos Estados son especializados en el sector agropecuario.

PALABRAS CLAVE: Agricultura Familiar, Crédito Rural, Región Norte.

\section{INTRODUÇÃO}

A agricultura sempre desempenhou um papel fundamental para a sobrevivência humana, porém, foi a partir do Século XVIII, com as mudanças no processo de desenvolvimento industrial e aumento populacional urbano, que a exploração agrícola passou a ter relevância capital para o desenvolvimento econômico do país, sobretudo na produção de alimento, energia, emprego, renda, mão de obra para a indústria e segurança alimentar, conforme visto no CODAF (2015)

Foi a partir da segunda Guerra Mundial (1939-1945) que teve inicio o processo de modernização da agricultura nos países em desenvolvimento, 
conhecido como "Revolução Verde", que, no final da década de 1960 e inícios de 1970, no Brasil, impulsionou a agricultura intensiva e possibilitou aumento da oferta de alimentos. Para isso, foi necessária a intervenção estatal através da concessão do crédito ao setor rural, um dos instrumentos importantes na expansão da atividade agropecuária, assim como todo um processo de abastecimento relacionamento a alimentação, segundo McMichael (2010).

No Brasil, o desenvolvimento agrícola e o crescimento da produção não significaram o aumento da população ocupada na agricultura, visto que a introdução de novas máquinas, equipamentos e também de insumos agrícolas contribuíram para o contínuo aumento da produtividade, mas também para o aumento do desemprego nesse setor, decorrente da própria mudança de produção agrícola, isto é, menos intensiva em mão de obra.

Com a abertura do mercado, no início dos anos de 1990, a crise na agricultura foi maior, pois com parcos recursos o setor não tinha suporte para atender a demanda e com isso perdeu competitividade no mercado internacional. Assim, o pequeno agricultor, desamparado pelas políticas de crédito agrícola, passou a reivindicar políticas de crédito diferenciadas através de movimentos sindicais dos trabalhadores rurais, como observado por Schneider et al. (2004).

Para atender as pressões dos agricultores, primeiramente, o Governo Federal criou o Programa de Valorização da Pequena Produção Rural - PROVAPE. Em 1996, foi criado o Programa Nacional de Fortalecimento da Agricultura Familiar - PRONAF, a fim de facilitar ao agricultor familiar empréstimos a juros baixos e o pagamento da dívida de longo prazo, que proporcionasse aumento de sua capacidade produtiva, elevação de renda, melhoria na qualidade de vida, além de promover sua permanência no campo e a dinamização do desenvolvimento do setor agrícola (MDA, 2011).

Inicialmente, as aplicações do crédito rural Pronaf ficaram concentradas nas regiões Sul e Nordeste, e, somente a partir do ano de 2000, o Programa abrangeu as demais regiões brasileiras, 
passando o Fundo Constitucional de Financiamento do Norte (FNO) a direcionar os recursos do crédito rural Pronaf aos agricultores familiares da região Norte (BANCO DA AMAZÔNIA, 2014)

Neste contexto, levantou-se o seguinte questionamento: - a aplicação da política de crédito rural dinamizou a agricultura familiar da região Norte brasileira?

Para dar resposta ao questionamento, esta pesquisa teve como objetivo geral analisar as aplicações dos recursos do FNO/Pronaf na região Norte. Mais, especificamente: i) analisar a evolução das aplicações do FNO/Pronaf na agricultura familiar na região em foco, no período de 2000 a 2010; ii) identificar quais os grupos familiares que têm demandado maior volume de recursos FNO/Pronaf dentro dos estados nortistas.

Nesse aspecto, a hipótese levantada foi a de que os recursos do crédito rural do FNO/Pronaf têm contribuído para que a Agricultura Familiar na região Norte seja mais dinâmica na região norte brasileira.
A expressão "Agricultura Familiar" passou a ser utilizada a partir da década de 1990, no campo das políticas públicas direcionadas ao setor agrícola. Lamarche (1998) conceitua exploração familiar como "uma unidade de produção agrícola onde propriedade e trabalho estão intimamente ligados à família".

No Brasil, segundo Schneider (2003), a agricultura familiar se consolidou a partir da criação Pronaf, em 1996. Para Bruno e Dias (2004), a política de créditos rurais criou condições favoráveis de financiamentos, e, quase sempre, assumiu o caráter de política de inclusão social ou de integração de grupos sociais economicamente carentes e desamparados nos mercados locais e regionais.

De acordo com o Censo Agropecuário de 2006, existem no Brasil cerca de: 4.367 .902 estabelecimentos familiares, o equivalente a $84,4 \%$ do total, com uma área ocupada de 80,25 milhões de hectares, ou seja, 24,3\% da área total (IBGE, 2010).

As políticas de créditos rurais implantadas pelo governo federal, de modo geral, privilegiaram as produções 
em larga escala voltadas para o mercado externo, deixando à revelia o pequeno agricultor (BASTOS, 2006).

No início dos anos 1990, o processo de abertura comercial e a desregulamentação do mercado afetaram a agricultura brasileira, submetendo-a a uma intensa concorrência com os países do Mercosul ${ }^{1}$ (SOUSA, 2009). Tal processo teve consequências negativas para os agricultores familiares que sem recursos ficaram expostos à concorrência externa, desamparados pelas políticas públicas do governo e tiveram que enfrentar o aumento da pobreza no campo (SILVA e MESQUITA, 2009).

A criação do Pronaf pelo Decreto 1.946/96 tornou-se um marco no processo de intervenção governamental na agricultura brasileira. O programa buscou apoiar e fortalecer o setor rural, além de promover o desenvolvimento regional, que tem o intuito de diminuir a pobreza no campo, com aumento da produção e geração de renda.

\footnotetext{
${ }^{1}$ O Mercado Comum do Sul - MERCOSUL é um tratado criado em 1991 com o objetivo de estabelecer um mercado comum entre os países acordados (Brasil, Argentina, Paraguai, Uruguai e Venezuela).
}

Em 2006 foi criada a Lei 11.326 da Agricultura Familiar, que trouxe um novo ajuste para seus beneficiários, ou seja, o empreendedorismo familiar rural. $\mathrm{Na}$ Amazônia, cabe ao Banco da Amazônia - BASA a gerência do FNO e cobre os estados do Acre (AC), Amapá (AP), Amazonas (AM), Pará (PA), Rondônia (RO), Roraima (RR) e Tocantins (TO), em nível de concessão de créditos para o setor primário.

\section{MATERIAL E MÉTODOS}

Esta pesquisa teve caráter exploratório com base em dados secundários, envolvendo o período de 2000 a 2010. As principais fontes utilizadas foram: Instituto Brasileiro de Geografia e Estatístico (IBGE) e Banco da Amazônia S.A. (BASA). Trata-se de um estudo comparativo dos recursos do crédito rural do FNO/Pronaf entre os estados da região Norte (Acre, Amapá, Amazonas, Pará, Rondônia, Roraima e Tocantins). Na verdade, a razão pela escolha desse Fundo se deu pela facilidade de acesso aos dados para uma melhor análise. 
Além das ferramentas de análises estatística descritivas como média $x^{-}=\frac{\sum_{i=1}^{n} x i}{n}, \quad$ desvio padrão $\sigma=\sqrt{\operatorname{Var}(x)}$ e coeficiente de variação $(\mathrm{CV})=\frac{(\sigma)}{(x)^{\prime}}$, foram utilizadas a Taxa de Variação $=\left(\left(\frac{V i}{V f}\right)-1\right) * 100$ e a Taxa Geométrica de Crescimento - TGC, baseada no modelo de Regressão Linear Simples log-linear, utilizando a seguinte regressão geral:

$$
\ln Y_{i t}=\alpha_{i}+b_{i} T+\epsilon_{t}
$$

Em que:

InYit = é o logaritmo natural da variável i, no ano t;

T = é uma variável tendência;

$\alpha_{i}=$ parâmetros que representa o valor médio da variável $i$;

$b_{i}=$ é o logaritmo natural da taxa geométrica de crescimento $(1+i)$, onde a taxa de crescimento $i$ é obtida do seguinte modo: $i=a n t i \ln b_{\mathrm{i}}-1 ; \mathrm{e}$,

$€_{\mathrm{t}}=$ é o tempo de erro aleatório. O ajustamento será feito por Mínimos Quadrados Ordinários MQO.

Utilizou-se, também, o Coeficiente de Correlação $\rho(x, y)=\frac{\operatorname{Cov}(x, y)}{\sigma x \sigma y}$, para medir o grau de relacionamento linear entre duas variáveis quantitativas. Seu valor situa-se entre -1 e 1 . 0 valor 0 (zero) significa que não há relação linear; o valor 1 indica uma relação linear perfeita e o valor -1 também indica uma relação linear perfeita, mas inversa. Quanto mais próximo estiver de 1 ou -1, mais forte é a associação linear entre as duas variáveis.

\section{RESULTADOS E DISCUSSÃO}

PRINCIPAIS CARACTERISTICAS DA REGIÃO NORTE DO BRASIL

Com 3,85 milhões de $\mathrm{km}^{2}$ de extensão territorial, equivalente a 45\% do território brasileiro, a região Norte possui uma população em torno de 15,86 milhões de habitantes, dos quais 4,19 milhões vivem na área rural, ou seja, 26\% do total da população (IBGE, 2010).

Em 2009, essa região registrou o Produto Interno Bruto $\left(\mathrm{PIB}^{2}\right)$ de mais de R\$ 163,21 milhões e sua participação relativa no PIB do país foi igual a 5,04\%. Seu PIB per capita ${ }^{3}$ foi da ordem de $R \$$ 10.583,94. A dinâmica dos setores são importantes para caracterizar a geração

\footnotetext{
2 PIB - Produto Interno Bruto - é o valor dos bens e serviços finais produzidos em uma economia em um dado período de tempo.

3 PIB per capita - é o Produto Interno Bruto dividido pela quantidade de habitantes de um país ou região.
} 
de riqueza e deveria haver um equilíbrio na produção desta entre as regiões, entretanto, Zander (2016) destaca que o setor agropecuária encontra-se em transformação e, apesar das políticas públicas (financiamentos, por exemplo), o governo deve criar políticas mais eficazes para um novo rural que se desenha, totalmente diferenciado do seu passado, mas que se ergue em termos de acumulação de riqueza, com destaque para o aumento da desigualdade regional.

Notadamente, o setor serviço foi o que apresentou maior participação no PIB regional (55\%), porém a variação registrada por esse setor $(41,14 \%)$, no período de 2000 a 2009, foi inferior à do setor agropecuário (44,60\%). Convém destacar que a relevância neste estudo recai sobre o setor agropecuário, em razão da AF está inserida nesta atividade.

Analisando-se os dados da lavoura temporária da região Norte, verificou-se que os produtos de maior participação na produção total foram: mandioca (27,77\%), abacaxi $(23,78 \%)$ e melancia $(13,76 \%)$. Juta e malva somente são produzidas nessa Região, com participação de quase 100\%.

A expansão das fronteiras agrícolas do Centro-Oeste para o Norte vem contribuindo para o aumento da participação da soja, especialmente nos estados do Pará e Tocantins. Esta cultura que em 2001 participava com menos de 1\% da produção brasileira, em 2010, participou com 2,4\% do total produzido no País.

Na verdade, esse tipo crescimento é importante para o desenvolvimento agrícola regional, principalmente, quanto ao aspecto de produção de oleaginosas para diversos fins (alimentícios e biodiesel). Embora a soja não entre no portfólio de produção da $A F$, mas pode surgir como opção de emprego no campo para aquele segmento.

Tudo isto, mostra a importância da fronteira agrícola para a produção de alimentos, que - no primeiro momento segue uma trajetória de apenas como lócus de produção de matérias - primas, tema já muito debatido por autores importantes, que estudam o Norte e a Amazônia brasileira e o seu papel na 
economia, como discutido por Domingues e Bermann (2012).

A produção da lavoura permanente regional, no período 2000 a 2010, teve a participação média de 7,6\% no total da lavoura permanente nacional. Entretanto, ambas não apresentaram crescimento no período em foco, haja vista que suas taxas geométricas de crescimento não foram significativas estatisticamente. Em 2010, a quantidade produzida pela lavoura permanente do país atingiu 30,93 milhões de toneladas, enquanto que a região Norte produziu cerca de 2,4 milhões de toneladas.

Nesse ano, em relação ao anterior, a produção elevou-se em cerca de 10\%. Linhas gerais, as lavouras permanentes são importantes na sustentação da AF nas áreas rurais, por que estas servem e/ou são utilizadas como um benefício para a formação de quintais que geram sombra, alimento e mesmo remédios para esta categoria que sobrevive de seu próprio sustento (FILGUEIRAS; HERRERA, 2012).

Em que pese uma redução geral da lavoura permanente, no Norte, ressaltase que existem algumas culturas que têm grande relevância no contexto da produção nacional, com maior participação, seja em quantidade ou em área, tais como: a pimenta-do-reino (83,86\%), dendê (cacho) (81,03\%), urucum (semente) (31,55\%), guaraná (sementes) (29,74\%), cacau (em amêndoas) (29,03\%), banana (cachos) (14,96\%) e coco da baía (mil frutos) (13,54\%). Observa-se, ainda, que a região Norte é a região que tem a maior produção de pimenta-do-reino e dendê, e a segunda maior produção de cacau (10 é o estado da Bahia). Destaca-se ainda, como a maior produtora de frutas tropicais do país, especialmente, açaí, taperebá, cupuaçu e bacuri.

Com relação à pecuária, no período de 2000 a 2012, houve considerável crescimento no efetivo bovino regional. No ano 2000, o rebanho contava com 24,51 milhões de cabeças e, em 2012, esse número elevou-se para 43,82 milhões, crescimento que ocorreu a uma TGC de 4,2\% a.a. No período, a produção média foi de 37,31 milhões, o que mostra a importância dessa atividade para a região, conforme Tabela 1 . 
Tabela 1. Efetivo da pecuária da Região Norte no período de 2000 a 2012.

\begin{tabular}{l|c|c|c|c|c|c|c}
\hline Ano & Bovino & Equino & Bubalino & Suíno & Ovino & $\begin{array}{c}\text { Galos, } \\
\text { frangas, } \\
\text { frangos e } \\
\text { pintos }\end{array}$ & Galinhas \\
\hline 2000 & 24.517 .612 & 580.641 & 700.084 & 2.619 .791 & 360.141 & 18.972 .976 & 10.304 .918 \\
2001 & 27.284 .210 & 592.192 & 709.837 & 2.630 .606 & 372.027 & 17.953 .082 & 9.641 .723 \\
2002 & 30.428 .813 & 595.375 & 699.731 & 2.187 .589 & 367.373 & 17.529 .974 & 9.062 .827 \\
2003 & 33.929 .590 & 633.976 & 722.299 & 2.163 .430 & 407.643 & 22.721 .489 & 9.512 .096 \\
2004 & 39.787 .138 & 661.833 & 705.726 & 2.083 .318 & 429.025 & 18.648 .006 & 9.324 .178 \\
2005 & 41.489 .002 & 668.057 & 728.004 & 2.100 .033 & 481.528 & 18.219 .721 & 9.255 .563 \\
2006 & 41.060 .384 & 670.439 & 706.072 & 1.962 .164 & 496.755 & 18.167 .075 & 9.501 .891 \\
2007 & 37.865 .772 & 682.411 & 704.424 & 1.739 .411 & 521.640 & 18.719 .344 & 9.472 .796 \\
2008 & 39.119 .455 & 695.865 & 720.287 & 1.629 .552 & 534.478 & 18.962 .485 & 9.482 .764 \\
2009 & 40.437 .159 & 712.700 & 714.852 & 1.627 .822 & 547.146 & 18.732 .113 & 9.074 .193 \\
2010 & 42.100 .695 & 736.075 & 752.830 & 1.607 .481 & 586.237 & 18.279 .456 & 9.511 .600 \\
2011 & 43.238 .310 & 775.398 & 820.133 & 1.569 .553 & 627.563 & 17.590 .910 & 9.828 .278 \\
2012 & 43.815 .346 & 756.604 & 808.883 & 1.489 .219 & 598.643 & 17.242 .993 & 9.548 .496 \\
\hline
\end{tabular}

Estatística Descritiva

\begin{tabular}{l|r|r|r|r|r|r|r}
\hline Média & 37313345,08 & 673966,62 & 730243,23 & 1954613,00 & 486938,38 & 18595355,69 & 9501640,23 \\
DPad & 6287436,85 & 62177,89 & 40091,20 & 385055,40 & 92442,79 & 1358579,92 & 321776,06 \\
CV & 593,46 & $1.083,93$ & $1.821,46$ & 507,62 & 526,75 & $1.368,73$ & $2.952,87$ \\
\hline
\end{tabular}

Taxa Geométrica de Crescimento

\begin{tabular}{l|c|c|c|c|c|c|c}
\hline TGC(\%) & $4,2^{\star}$ & $2,37^{\star *}$ & $\begin{array}{c}1,03^{*} \\
(3,776)\end{array}$ & $\begin{array}{c}-4,69^{*} \\
(-14,038)\end{array}$ & $\begin{array}{c}5,03^{*} \\
(16,890)\end{array}$ & $\begin{array}{c}-0,563 \text { n.s } \\
(-1,130)\end{array}$ & $\begin{array}{c}-0,139 n . s \\
(-0,544)\end{array}$ \\
\hline
\end{tabular}

Fonte: Elaborada a partir dos dados do IBGE/Produção Pecuária Municipal - PPM (2013).

Nota: $\left(^{\star}\right)$ e $\left(^{(\star}\right)$ significância estatística a 1\% e 5\%; n.s. não significativa.

O rebanho de equinos evoluiu a uma TGC de 2,37\% a.a., registrando a média anual de 673.966 cabeças. Já o rebanho suíno apresentou uma redução a uma TGC de $-4,69$ a.a., no período em foco. A criação de ovinos cresceu a uma taxa de 5,03\% a.a., no mesmo período. Aves e galinhas apresentaram uma taxa de $-0,56$
$\%$ e $-0,14 \%$ a.a., respectivamente. Esta atividade da pecuária, como bem destaca Santos et al (2017) serve como reserva de valor para a $A F$, que pode disponibilizar recursos mais imediatos quanto necessita e, nesse aspecto, o crédito tem sido uma opção para a pecuária dos pequenos agricultores e AF. 
PERFIL DA AGRICULTURA FAMILIAR (AF)

DA REGIÃO NORTE

Por ocasião do Censo Agropecuário de 2006, a AF da região Norte era composta por 413.101 estabelecimentos, equivalente a 9,46\% do total nacional. Estes ocupavam uma área em torno de 16,65 milhões de hectares, ou seja, 20,80\% da área ocupada pela AF em nível nacional (Tabela 2). Já a $A N F^{4}$ possuía 62.674 estabelecimentos e ocupava uma área de 38,14 milhões de hectares, o que representava $7,76 \%$ e 15,27\%, respectivamente, em relação ao total do Brasil (Tabela 2).

No que tange a pecuária na AF regional, o destaque é para a produção de leite de vaca e o seu maior produtor é o estado de Rondônia com mais de 512,33 milhões de litros, seguido pelo Pará e Tocantins com 298,53 e 104,80 litros, respectivamente, quantidades que mostram a pouca participação da atividade pecuária na AF. Observa-se, que estes dados só são possíveis de

4 Segundo IBGE os estabelecimentos não enquadrados na classificação da Lei 11.326/2006, foram designados como Agricultura Não Família ANF. análise, mediante $\mathrm{O}$ Censo da AF, elaborado pelo IBGE (2010).

PARTICIPAÇÃO DO FINANCIAMENTO DO CRÉDITO RURAL FNO/PRONAF NA REGIÃO NORTE

No período de 2000 a 2010, em que - FNO passou a operacionalizar os recursos do Pronaf, observou-se que o crescimento ocorreu de forma gradual, mas consecutiva entre os estados da Região. Os valores aplicados foram em torno de $R \$ 3,22$ bilhões, dos quais 48,79\% foram demandados pelo o estado Pará. Os demais estados da região tiveram a seguinte distribuição: Rondônia (17,14\%), Tocantins (15,19\%) e Amazonas (9,22\%), Acre (5,26\%), Roraima (2,50\%) e Amapá (1,91\%) do total dos recursos. Observou-se que os três últimos estados relacionados tiveram pequena participação nos créditos disponibilizados para agropecuária.

Finalmente, para se destacar a importância do crédito FNO/Pronaf no fortalecimento da produção agropecuária da agricultura familiar foram calculados os coeficientes de correlação (Tabela 3). Os resultados 
mostraram uma relação positiva entre o FNO/Pronaf e o PIB da região Norte em torno de 70\%, assim como do FNO/Pronaf e a quantidade produzida da lavoura temporária e o Valor Bruto da Produção - VBP do Norte que foi mais de $83 \%$ e $92 \%$, respectivamente.

No entanto o FNO/Pronaf, em relação à quantidade da lavoura permanente e VBP, registrou relação negativa. Concluiu-se que os recursos do crédito são direcionados em grande parte para a produção das lavouras temporárias, devido sua importância na produção de alimentos, mais rápido retorno e dinâmica na transação desses produtos. Assim, as discussões da disponibilidade dessa linha de crédito (PRONAF) são obtidas junto ao Banco da Amazônia e, por isso, é uma análise mais quantitativa.

Tabela 2. Número de estabelecimentos, área (ha) e pessoal ocupado (PO) da AF e ANF na Região Norte: 2006

\begin{tabular}{c|c|c|c|c|c|c}
\hline \multirow{2}{*}{ UF } & \multicolumn{4}{|c|}{ AF } & \multicolumn{3}{c}{ ANF } \\
\cline { 2 - 7 } & Estabelecimentos & Área (ha) & PO na AF & Estabelecimentos & Área (ha) & PO na ANF \\
\hline Total Norte & 413.101 & 16.647 .328 & 2.580 .392 & 62.674 & 38.139 .968 & 518.565 \\
\hline Total Brasil & 4.367 .902 & 80.250 .453 & 23.734 .815 & 807.587 & 249.690 .940 & 8.337 .972 \\
\hline Norte /Brasil & 9,45 & 20,74 & 10,87 & 7,76 & 15,27 & 6,22 \\
\hline
\end{tabular}

Fonte: Dados do IBGE/Censo Agropecuário 2006 - Agricultura Familiar.

Tabela 3. Correlação entre FNO/Pronaf, PIB, Qtde LTemp VBPLtemp, Qte_Lperm_Norte,VBP_Lperm da região Norte no período de 2000 a 2009.

\begin{tabular}{|c|c|c|c|c|c|c|}
\hline & FNO/Pronaf & $\mathrm{PIB}$ & Qte_Ltemp & VBP_Ltemp & Qte_Lperm & VBP_Lperm \\
\hline FNO/Pronaf & 1 & & & & & \\
\hline $\mathrm{PIB}$ & 0,70 & 1 & & & & \\
\hline Qte_Ltemp & 0,83 & 0,87 & 1 & & & \\
\hline VBP_Ltemp & 0,93 & 0,78 & 0,88 & 1 & & \\
\hline Qte_Lperm & $-0,06$ & $-0,19$ & $-0,12$ & $-0,20$ & 1 & \\
\hline VBP_Lperm & $-0,30$ & $-0,25$ & $-0,49$ & $-0,30$ & 0,39 & 1 \\
\hline
\end{tabular}

Fonte: Elaborado a partir dos dados do IBGE (2012) Produção Agrícola Municipal, PIB regional, e dados do FNO/Pronaf, obtidos através da Gerência de Agricultura Familiar - GAF, BASA (2010). 


\section{CONCLUSÃO}

De modo geral, a AF na região Norte representa importante contribuição para o segmento agrícola, mas que necessita de incentivos de políticas públicas mais eficientes para proporcionar não só acesso ao crédito, mas também conhecimento técnico aos agricultores familiares para que possam dinamizar sua produtividade, pois em sua maioria, ainda utilizam formas de produção rudimentar.

O que se percebe por meio dos dados do Censo Agropecuário de 2006 é que a grande concentração de terras para cultivo existe tanto no Brasil quanto na região Norte, haja vista que o número de estabelecimentos da agricultura familiar é cinco vezes maior que o da agricultura não familiar e aquela ocupa, apenas, um terço da área ocupada por esta. Isto aponta para a necessidade de políticas públicas diferenciadas como é o caso do Pronaf bem como uma política mais dinâmica da Reforma Agrária em curso a longo tempo no Brasil, o que Zander (2016) chama a atenção para uma mudança no perfil da estrutura agrária em curso.
Ademais, existem entraves para maior dinamismo é o fato de que a política de financiamento do Pronaf ainda apresenta exigências que dificultam - acesso do agricultor ao crédito. Analisando a evolução das aplicações do FNO/Pronaf na agricultura familiar dos estados da região Norte, observou-se que, no período de 2000 a 2010, em que - FNO passou a operacionalizar os recursos do PRONAF, as aplicações desses recursos evoluíram de forma gradual, mas consecutiva na região.

Os valores aplicados foram em torno de $R \$ 3,22$ bilhões, dos quais 48,79\% foram demandados pelo o estado Pará, ficando o restante distribuído pelos estados de Rondônia (17,14\%), Tocantins (15,19\%), Amazonas (9,22\%), Acre (5,26\%), Roraima (2,50\%) e Amapá $(1,91 \%)$. Nos três últimos estados em que a participação nos créditos disponibilizados é pequena, o setor agropecuário apresentou-se pouco dinâmico.

Admite-se que o crédito tem contribuído para o volume maior de produção, tanto assim que a correlação entre crédito e valor da produção 
agropecuária dos estados foi positiva. Em quase todos os estados, o FNO/Pronaf apresentou baixa correlação positiva na quantidade produzida da lavoura temporária e negativa na quantidade produzida da lavoura permanente, assim como no Valor Bruto da Produção (VBP).

Apenas os estados do Pará e Tocantins apresentaram alta correlação do PIB com a lavoura temporária, que foi a que mais contribuiu para o PIB da Região, uma vez que é nela que se produzem os produtos da cesta básica de alimentos. Isto vem confirmar a hipótese do trabalho de que o crédito tem contribuído para a dinâmica da produção agropecuária no Norte, ainda que existam entraves de diversas ordens.

Nesse contexto, portanto, a agricultura familiar é extremamente importante para o desenvolvimento regional, haja vista sua contribuição para a produção de subsistência e, também, para o mercado de produtos alimentares, contribuindo para a garantia da segurança alimentar no país. No âmbito geral, na região Norte apresentou-se dinâmica no que se refere à agricultura tradicional, principalmente na produção da lavoura temporária com maior destaque para a mandioca, abacaxi e melancia. E, ainda, no setor pecuário, destaca-se o crescimento do rebanho bovino.

No entanto, não se pode afirmar que os recursos do FNO/Pronaf têm sido de grande relevância para o crescimento da produção da AF como um todo, pela impossibilidade em classificar o que é AF, da não familiar. Não há dados oficiais que comprovem essa separação, pelo menos até o momento da pesquisa, uma vez que o IBGE trouxe esse recorte apenas para o Censo Agropecuário de 2006. Além disso, o tempo (11 anos), em que o FNO passou a atender o Norte, é pouco para que seja feita uma boa avaliação da aplicação dos financiamentos na Região.

Cabe destacar, também, que os estados como Pará, Rondônia e Tocantins têm contribuído para a demanda desses recursos e a própria dinâmica do programa, que promove adaptações a cada ano agrícola, seja na criação ou simplificação de grupos familiares. Isso vem mostrando a importância do PRONAF como política 
pública, como é o caso dos grupos familiares Pronaf AF e Pronaf Mais Alimentos, que nos últimos três anos apresentou significada demanda para o financiamento de infraestrutura do sistema de agricultura familiar.

Mas não se deve esquecer que ainda há muito a se melhorar para atender uma parcela de agricultores que ainda encontram-se excluídos do processo do crédito rural, por inúmeras razões que vão do desconhecimento até o medo de contrair dívidas. De fato, o PRONAF ainda que com todas as dificuldades para atender os beneficiários do programa, está sendo positivo uma vez que iniciou no ano de 2000 com um volume de $R \$$ 130 milhões e, em 2010, esse valor passou para $\mathrm{R} \$ 488,55$ milhões, ou seja, uma variação de 275,29\% nos 11 anos.

\section{REFERÊNCIAS}

BANCO DA AMAZÔNIA. Relatório de Sustentabilidade, 2014. Banco da Amazônia S/A. Disponível: $<$ http://bancoamazonia.com.br/index.ph p/component/jdownloads/finish/98relatorio-2014/1250-relatorio-desustentabilidade-2014?!temid=0>. Acesso em: 10 Jan. 2017.

BASTOS, F. Ambiente institucional no financiamento da agricultura familiar. São
Paulo. São Paulo: Polis; Campinas: CERES

- Centro de Estudos Rurais do IFCH, UNICAMP, 2006.

BRUNO, R. Q. L.; DIAS. M.M. As politicas públicas de crédito para os assentados rurais no Brasil. Relatório de Consultoria. Rio de Janeiro, 2004.

COMPETÊNCIAS DIGITAIS PARA AGRICULTURA FAMILIAR - CODAF. A importância da Agricultura Familiar. UNESP, $2015 . \quad$ Disponível: $<$ http://codaf.tupa.unesp.br/agriculturafamiliar/a-importancia-da-agriculturafamiliar>. Acesso: 30 Mar. 2017.

DOMINGUES, M. S.; BERMANN, C. O arco de desflorestamento na Amazônia: da pecuária à soja. Ambiente e Sociedade, São Paulo, v. 15, n. 2, Mai./Ago., 2012.

FILGUEIRAS, G. C.; HERRERA, J. A. O segmento da agricultura familiar no estado do Pará: um olhar a partir dos dados do Censo de 2006. Cadernos CEPEC, Belém, v. 1, n. 7, Jun., 2012.

FRANÇA, C. G, DEL GROSSI, M. E, MARQUES, V. P. M. A. Brasília: MDA, 2009. Disponível em: $<$ mineiropt.com.br/arquivosestudo/arq4b 10179787f8b.pdf>. Acesso em: 27 Mai. 2012.

HADDAD, P. R. Medidas de localização e de especialização. In: HADDAD, P. R. et al. (Org.). Economia regional: teorias e métodos de análise. Fortaleza: BNBETENE, 1989. 
HÉBETTE, J. Cruzando a fronteira: 30 anos de estudo do campensinato na Amazônia. Belém: ADUFPA, 2004.

INSTITUTO BRASILEIRO DE GEOGRAFIA E ESTATÍ́STICA - IBGE. Censo Agropecuário, 2006.

LAMARCHE, H. (Coord.). Agricultura familiar: do mito à realidade. Campinas, Unicamp, 1998.

LEITE, S. P. Reforma Agrária como estratégia de desenvolvimento: uma abordagem a partir de Barraclough, Furtado, Hirschman e Sem. Boletim de Ciências Econômicas XLX. 2007.

MCMICHAEL, P. The World Food Crisis in Historical Perspective. In: MAGDOFF, F. e TOKAR, B. Agriculture and Food in Crisis: conflict, resistance, and renewal. Nova Iorque: Monthly Review Press, 2010.

MATTEI, L. Impactos do Pronaf: análise de indicadores. Ministério do Desenvolvimento Agrário, Núcleo de Estudos Agrários e Desenvolvimento Rural. Brasília, 2005.

MINISTÉRIO DO DESENVOLVIMENTO AGRÁRIO - MDA. O Selo Combustível Social, 2011. Disponível em: <http://www.mda.gov.br/sitemda/secreta ria/saf-biodiesel/o-selo-com

bust\%C3\%ADvel-

social\#sthash.F5xeBIGi.dpuf>. Acesso em: 10 Jan. 2017.

SANTOS, M. A. S.; LOURENÇO JÚNIOR, J. B.; SANTANA, A. C.; HOMMA, A. K. O.; ANDRADE, S. J. T.; SILVA, A. G. M. Caracterização do nível tecnológico da pecuária bovina na Amazônia Brasileira.
Revista de Ciências Agrárias., v. 60, n. 1, p. 103-111, jan./mar., 2017.

SCHNEIDER, S. Teoria Social, Agricultura Familiar e Pluriatividade. Revista Brasileira de Ciências Sociais, v. 18, n. 51, Fev., 2003.

SILVA, J. R. S., MESQUITA, B. A. Algumas consequências da política neoliberal para aagricultura familiar na Amazônia brasileira: exclusão social e concentração de riqueza. In: ENCUENTRO DE GEÓGRAFOS DE AMÉRICA LATINA, 12., 2009. Anais... Uruguai: Montevidéu, 2009.

SILVA, R. P. Avaliação do Desempenho dos Principais Produtos da Pauta de Exportação do Agronegócio Brasileiro no Período de 1997 a 2008. Dissertação (Mestrado em economia). Universidade Federal do Pará, Belém, 2011.

SOUSA, J. M. P. Avaliação do financiamento da agricultura fa miliar na produção, ocupação e renda. (Série BNB Teses e Dissertação, 18). Fortaleza: Banco do Nordeste do Brasil, 2009.

ZANDER, N. O mundo rural no novo século (um ensaio de interpretação). In: VIEIRA FILHO, J. E.; GASQUES, J. G. (Org.) Agricultura, transformação produtiva e sustentabilidade. Brasília: ABAG/IPEA, 2016. 